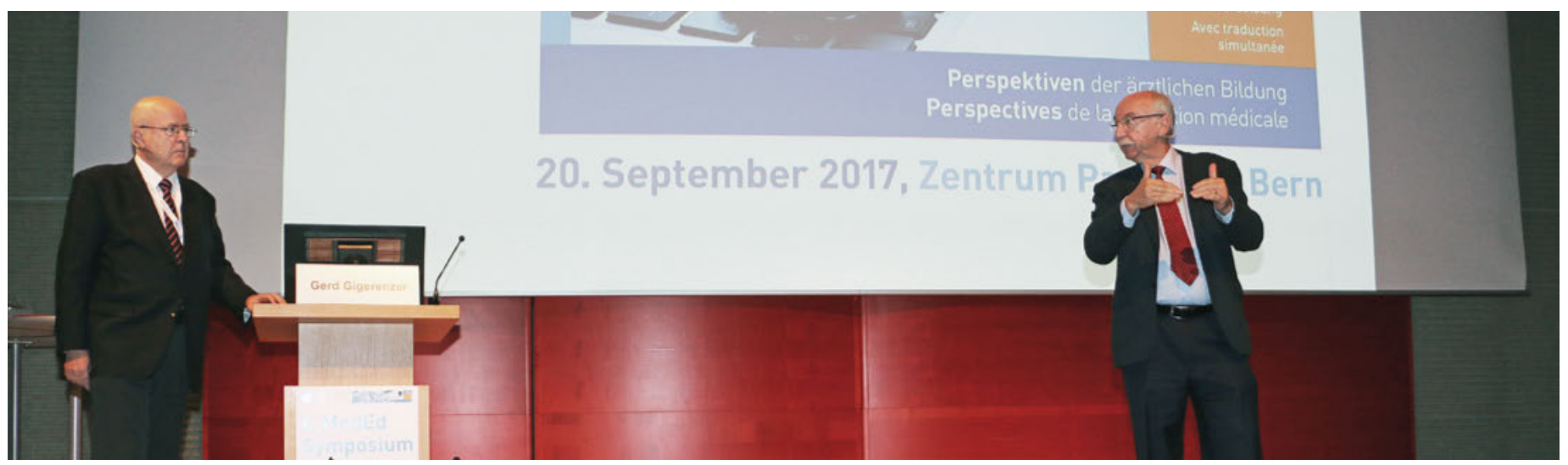

Le quatrième symposium MedEd a été l'occasion d'un échange intense sur les perspectives de la formation médicale: Werner Bauer (à gauche), président de I'ISFM et organisateur de la manifestation, en discussion avec Gerd Gigerenzer, conférencier.

\title{
Nouvelles technologies, nouvelles compétences, nouvelles méthodes
}

\section{Bruno Kesseli}

Dr méd. et lic. phil, rédacteur en chef

La quatrième édition du symposium MedEd de l'Institut suisse pour la formation médicale postgraduée et continue (ISFM) s'est déroulée pour la première fois au Centre Paul Klee et a une fois de plus proposé un programme de qualité: des exposés passionnants et instructifs, des séminaires d'approfondissement, une interview et une table ronde en guise de conclusion ont suscité des discussions intéressantes et présenté des pistes de réflexion utiles aux participants.

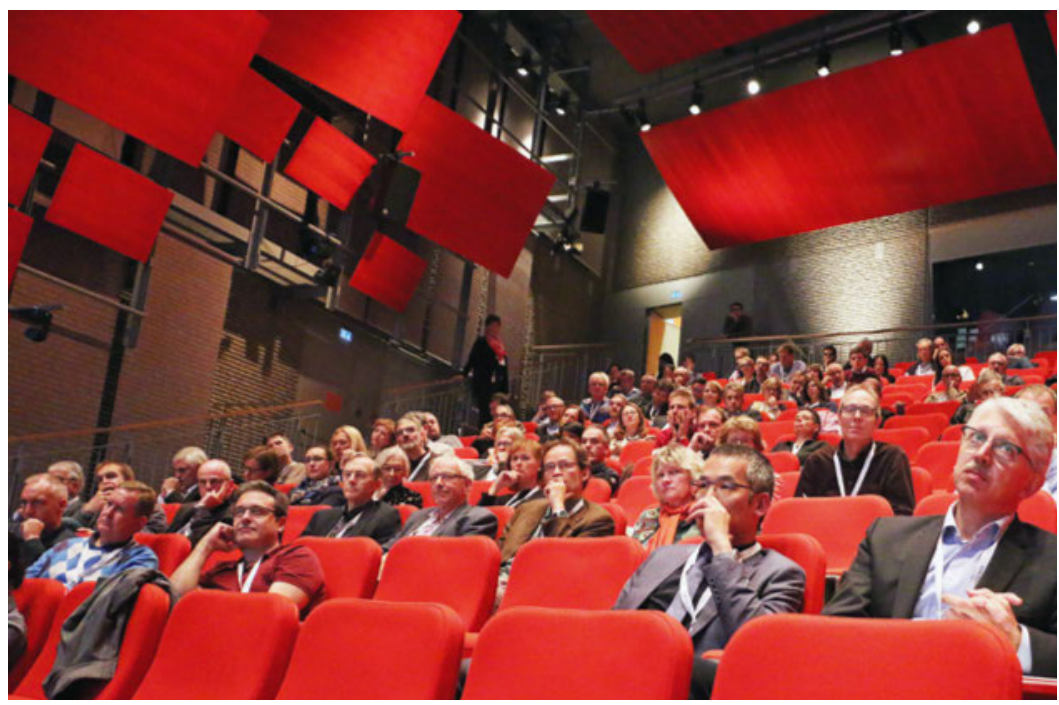

Intéressé et ouvert à la discussion: le public au symposium MedEd.
L'incertitude qui accompagne souvent les médecins dans leurs prises de décision a été un thème souvent abordé lors du symposium. Un doute a cependant pu être éliminé d'entrée. Les invités de la table ronde ont assisté sur écran géant à l'élection d'un médecin au Conseil fédéral en la personne d'Ignazio Cassis. Evènement rare, comme l'a rappelé Werner Bauer, président de l'ISFM, dans son discours d'ouverture. Ignazio Cassis n'est que le deuxième médecin à siéger au gouvernement fédéral. Le mandat de son collègue Adolf Deucher, membre du même parti et précurseur de la SUVA, remonte à plus d'un siècle. Elu en 1883, le Thurgovien est mort en 1912 en fonction, à l'âge de 81 ans.

Le symposium MedEd n'est cependant pas consacré aux questions politiques, mais aux «perspectives de la formation médicale». Pour Werner Bauer, la philosophie et l'ambition de l'ISFM visent à ne pas seulement gérer 
la formation médicale, mais à la façonner et à contribuer activement à son amélioration. Dans ce concept, le symposium MedEd joue un rôle important en tant que forum d'échange pour les spécialistes de la formation médicale. La reconnaissance et l'analyse des développements qui se répercutent sur la formation médicale sont des éléments clés de la manifestation. Pour la quatrième édition, l'accent a été mis sur les nouvelles technologies, les nouveaux objectifs de formation et compétences et les méthodes didactiques qui y sont associées, a précisé Werner Bauer.

\section{La pertinence de la formation médicale}

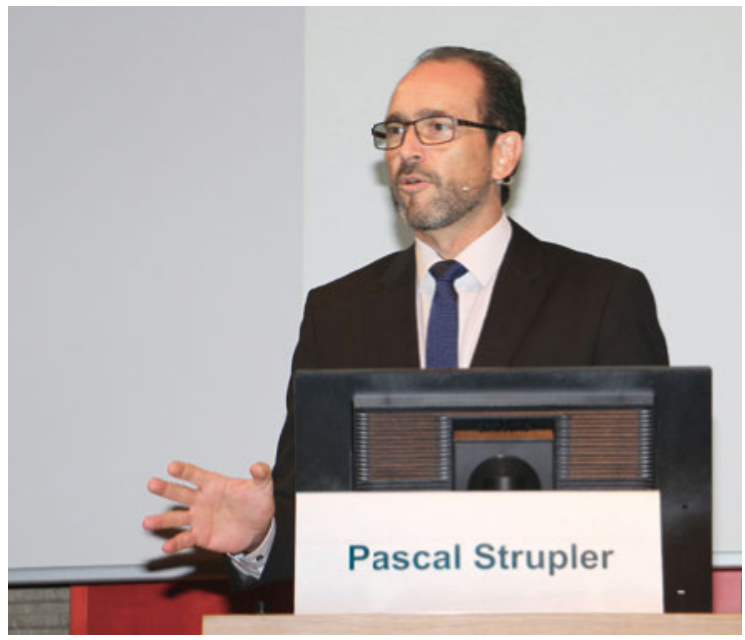

"Va où le puck sera»: Pascal Strupler, directeur de l'OFSP.

Les nouvelles technologies, résumées sous le thème de la numérisation, ont d'emblée été abordées par Pascal Strupler, premier orateur. Le directeur de l'Office fédéral de la santé publique est un habitué du symposium MedEd. Dans cette tradition, il a adressé son discours de bienvenue aux participants. Pascal Strupler a ensuite rappelé que le développement technologique et la numérisation ne se répercutent pas seulement sur la formation médicale, mais sur l'ensemble du système de santé. Il a également souligné qu'il était fondamental de réfléchir aux attentes liées aux nouveaux développements technologiques et aux perspectives que cela implique et, précisé que la formation médicale, dont la pertinence pour le système de santé ne saurait être suffisamment appréciée, devait être aménagée avec anticipation et clairvoyance. Il a aussi mentionné la plate-forme de l'OFSP «Avenir de la formation médicale» en place depuis 2010, qui permet au corps médical de participer à la discussion. Il a terminé son exposé en citant Wayne Gretzky, hockeyeur de classe mondiale: «Patine vers l'endroit où le puck ira, et non vers là où il a été.»

\section{L'informatique en progression}

L'informatique va-t-elle dépasser la formation médicale (continue)? C'est sous ce titre qu'était placé l'exposé de Christian Lovis [1]. Pour ce qui concerne la légèreté de sa présentation à la fois divertissante et amusante, le Professeur d'informatique médicale de l'Université de Genève et directeur du département des sciences de l'information médicale aux HUG s'est inscrit dans la tradition anglo-saxonne de personnalités telles que Steve Jobs ou Bill Gates. "Nous sommes passés de l'époque de la lenteur à celle de la rapidité», a-t-il constaté. Une évolution qui ne pourra pas être inversée. Dans le domaine de l'analyse de gros volumes de données, les machines ont atteint une vitesse que l'homme n'arrive pas à suivre. Ainsi, le logiciel Watson développé par IBM intègre les données de 30 milliards de radiographies, un volume d'informations inimaginable à l'échelle humaine. Christian Lovis a constaté que les médecins n'étaient pas du tout ou insuffisamment formés dans le domaine de l'intelligence artificielle et du Big Data. Une nécessité pourtant, car les patients mettent de plus en plus souvent les médecins au défi. A ce propos, Christian Lovis a renvoyé à l'auteur américain Eric Topol, qui a décrit dans des ouvrages tels que The Patient will see you now ou The Creative Destruction of Medicine le potentiel révolutionnaire d'une médecine numérisée. Les médecins doivent donc être préparés au fait que l'information médicale ne sera plus seulement accessible à une minorité. «L'informatique peut être utile», a-t-il résumé, mais il n'a laissé aucun doute sur le fait qu'à l'avenir, il faudra lui accorder une place plus importante dans la formation médicale.

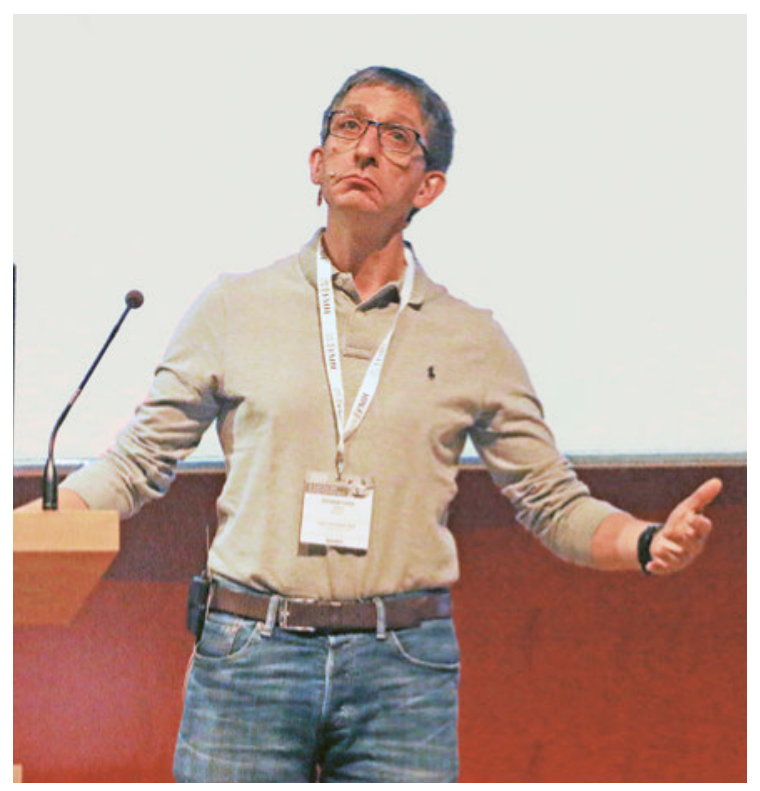

Le flegme britannique: Christian Lovis. 


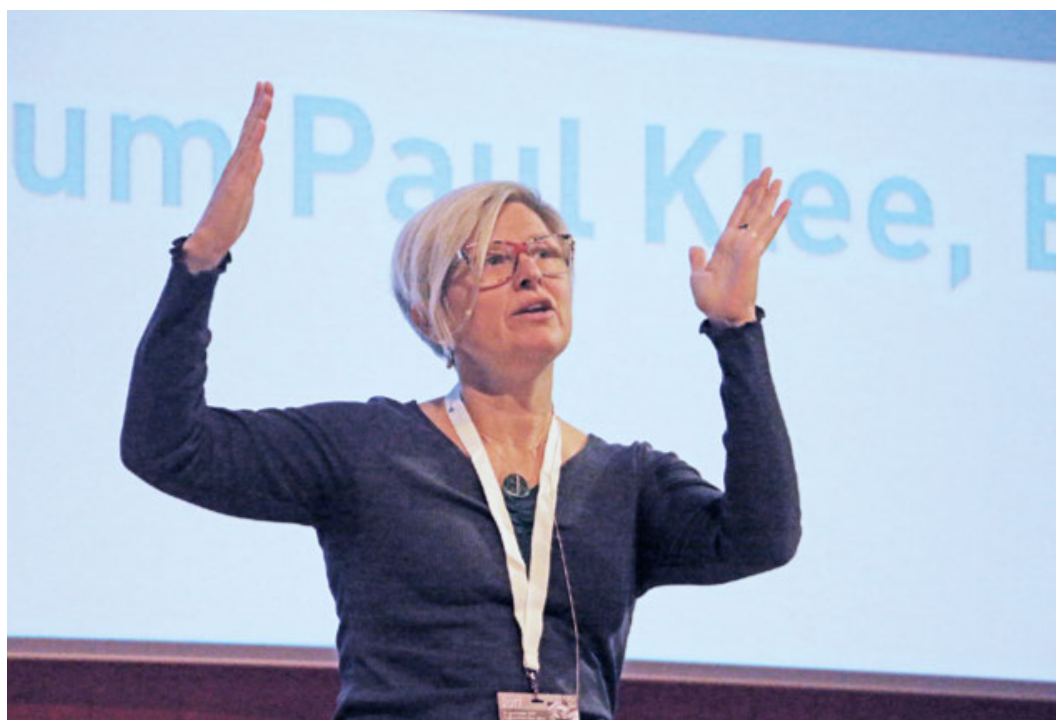

La communication est une compétence essentielle du médecin: Tanja Krones.

\section{Patients simulés et concept de Blended Learning}

Si Christian Lovis a dessiné les grandes lignes des développements dans la technologie de l'information, Tanja Krones, Professeur et médecin adjointe d'éthique clinique à l'Institut d'éthique biomédicale et d'histoire de la médecine de l'Université de Zurich, s'est focalisée sur le bénéfice de méthodes concrètes dans la formation médicale postgraduée et continue. Elle a mis l'accent sur les compétences de communication, les processus d'équipe interprofessionnels et un transfert efficace du savoir et des compétences. La communication, a-t-elle souligné, est une compétence médicale fondamentale qui n'est pas naturelle, mais qui doit être acquise et peut être améliorée tout au long de la vie. L'apprentissage des compétences de communication est le plus facile dans des scénarios proches du quotidien clinique et avec un feed-back professionnel. Tout cela doit aussi tenir compte des processus d'équipe interprofessionnels. De son point de vue, les patients et scénarios simulés sont une méthode clé pour acquérir des compétences de communication. Quant à la critique souvent exprimée selon laquelle il s'agirait de patients simulés «factices», elle la considère comme injustifiée. Elle s'est dite convaincue que les critiques n'ont probablement jamais assisté à un entraînement simulé.

Tanja Krones a poursuivi en soulignant que l'apprentissage en ligne est un outil utile pour le transfert du savoir et de compétences. Notamment lorsque l'apprentissage en ligne s'effectue dans le cadre de concepts combinés tels que le Blended Learning ou le Flipped classroom. Toutefois, ces nouveaux outils ne doivent pas remplacer les formes d'apprentissage classiques, mais être considérés comme des options d'apprentissage supplémentaires. Tanja Krones a illustré son propos avec de nombreux exemples tirés de la pratique de l'Hôpital universitaire de Zurich. Le débat animé au terme de son exposé a montré qu'il a suscité un vif intérêt parmi le public.

\section{«D'énormes déficits»}

Dans son exposé intitulé «Décider dans l'incertitude», Gerd Gigerenzer a démontré de façon impressionnante comment les données biostatistiques peuvent servir à semer la confusion et à manipuler, mais aussi à établir la transparence et la compréhension. Le directeur au centre Harding de compétences en matière de risques de l'Institut Max Planck pour la recherche pédagogique de Berlin s'est fait un nom dans le monde entier en tant qu'expert dans le domaine de la compétence en matière de risques. Il considère qu'à notre époque, la capacité d'évaluer correctement les risques est aussi importante que l'était la capacité de lire et écrire il y a 150 ans. En médecine, une appréciation réaliste des risques n'est pas facile, notamment parce que les données d'études sont souvent maquillées pour les faire apparaître sous un meilleur jour. Généralement, cela cache d'énormes intérêts. Gerd Gigerenzer a montré la différence entre une communication en matière de risques trompeuse et transparente au moyen de paramètres connus: taux de survie, risque relatif et probabilité conditionnelle. Il a montré avec une clarté surprenante comment, dans le cadre de programmes de dépistage, on triche avec des taux de survie à cinq ans, des surdiagnostics et une réduction relative du risque. La publicité pour les médicaments aime aussi mettre l'accent sur la réduction relative du risque. Ainsi, lorsqu'un hypolipidé-

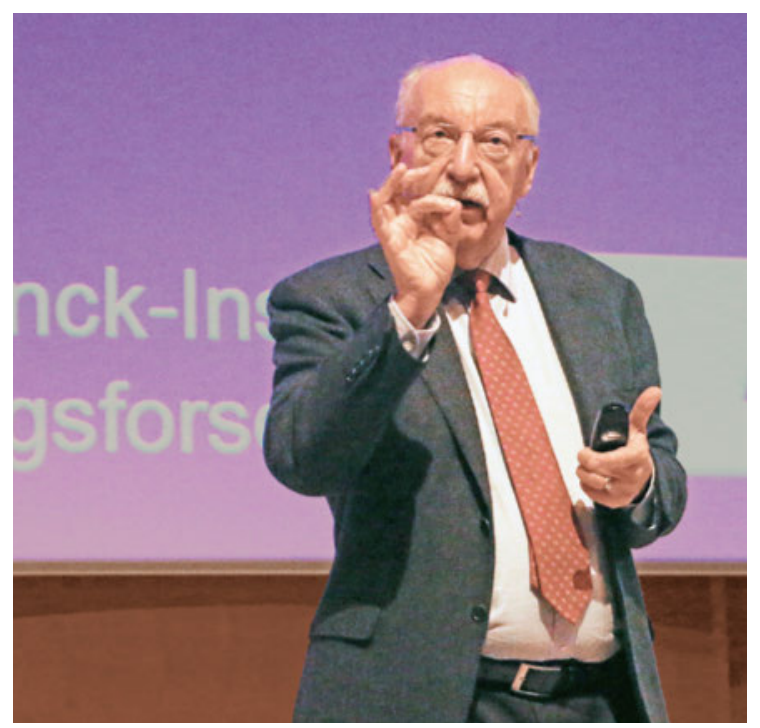

«Penser n'est pas un soft skill»: Gerd Gigerenzer. 
miant réduit le risque d'AVC de $48 \%$, cela paraît très convaincant. En clair, cela signifierait dans le cas concret une réduction de 2,8 à 1,5 cas sur 100 patients, ce qui est nettement moins impressionnant.

Comme l'a montré Gerd Gigerenzer, le corps médical souffre d'importants déficits lorsqu'il s'agit d'analyser des données biostatistiques. Beaucoup de médecins se laissent donc assez facilement duper. Dans ce domaine, la formation (prégraduée) médicale présente un grand potentiel d'amélioration. Pour Gerd Gigerenzer, la compétence en matière de risques est un élément essentiel de la révolution numérique. Il s'est dit convaincu qu'il est essentiel d'apprendre aux étudiants en médecine à penser: «Penser n'est pas un soft skill».

\section{Trois ateliers et une interview}

Comme la tradition le veut, l'après-midi du symposium s'est ouvert avec trois ateliers parallèles [1] dans le cadre desquels les thèmes suivants ont été abordés et discutés:

- Intelligence artificielle et formation médicale: quel avenir? [2]

- Nouvelles méthodes dans la formation postgraduée: Nice to have or need to have? [3]

- Médecin et après? Les enjeux du choix de carrière. [4]

Ensuite, toute l'attention s'est portée sur les médecins en formation postgraduée. Werner Bauer a invité Livia Dülli et Rolf Erlebach à le rejoindre sur scène pour une interview. Tous deux travaillent actuellement comme médecins-assistants à l'Hôpital cantonal de Winterthour. Livia Dülli en pédiatrie, Rolf Erlebach en anesthésie. Ils ont constaté qu'une grande importance était accordée à la formation postgraduée dans leurs services respectifs, que l'ambiance y était favorable à la formation postgraduée, même si chaque établissement de

\section{ISFM-Award 2017: les lauréats}

Le quatrième symposium MedEd a été I'occasion de révéler le nom des lauréats de I'ISFM-Award 2017 pour un engagement exceptionnel dans la formation postgraduée. Le prix est décerné sur la base de la nomination par d'anciens médecins en formation. Cette année, les formateurs suivants ont reçu une distinction:

Dr méd. Frank Bochmann, Lucerne; Dr méd. Johanna Büchel, Bâle; Dr méd. Christian Candrian, Lugano; PD Dr méd. Heiko Frühauf, Zurich; Dr méd. Andreas Geiser, Schlieren; Dr méd. Andrea Grêt, Soleure; Prof. Dr méd. Andreas Günthert, Lucerne; méd. pract. Ann-Kristin Hörsting, Winterthour; Dr méd. Lionel Jacquier, Martigny; Dr méd. Rachida Marir, Lausanne; Dr méd. Luzia Meier, Bâle; Prof. Dr méd. Dr sc. nat. Alexander A. Navarini, Zurich; PD Dr méd. Cäcilia Reiner, Zurich; Dr méd. Diego San Millán, Sion; Dr méd. Thomas Sauter, Berne; PD Dr méd. Parham Sendi, Bâle; Dr méd. Christophe Sénéchaud, Saint-Imier; Dr méd. Philipp Stillhard, Coire; Dr méd. Birgitta Thomann, Schlieren; Prof. Dr méd. Stephan Vavricka, Zurich; PD Dr méd. Mathias Worni, Berne.

formation postgraduée présente des difficultés spécifiques. Les exemples qu'ils ont cités ne se rapportaient pas à leur poste de travail actuel, mais concernaient des difficultés souvent discutées avec les collègues de travail. Ainsi, les revendications en matière de formation postgraduée sont difficiles à imposer lorsqu'elles entrent en conflit avec la durée de travail prescrite ou qu'elles doivent être intégrées dans des services où la planification est difficile, comme par exemple au service des urgences. Un aspect négatif récurrent qu'ils ont cité est la charge de travail administratif croissante au détriment du temps disponible pour le contact direct avec les patients. De plus, ils ont fait part de leur désir de bénéficier de plus d'enseignement au chevet du patient et souligné l'importance du feedback de la part de collègues expérimentés. Les deux

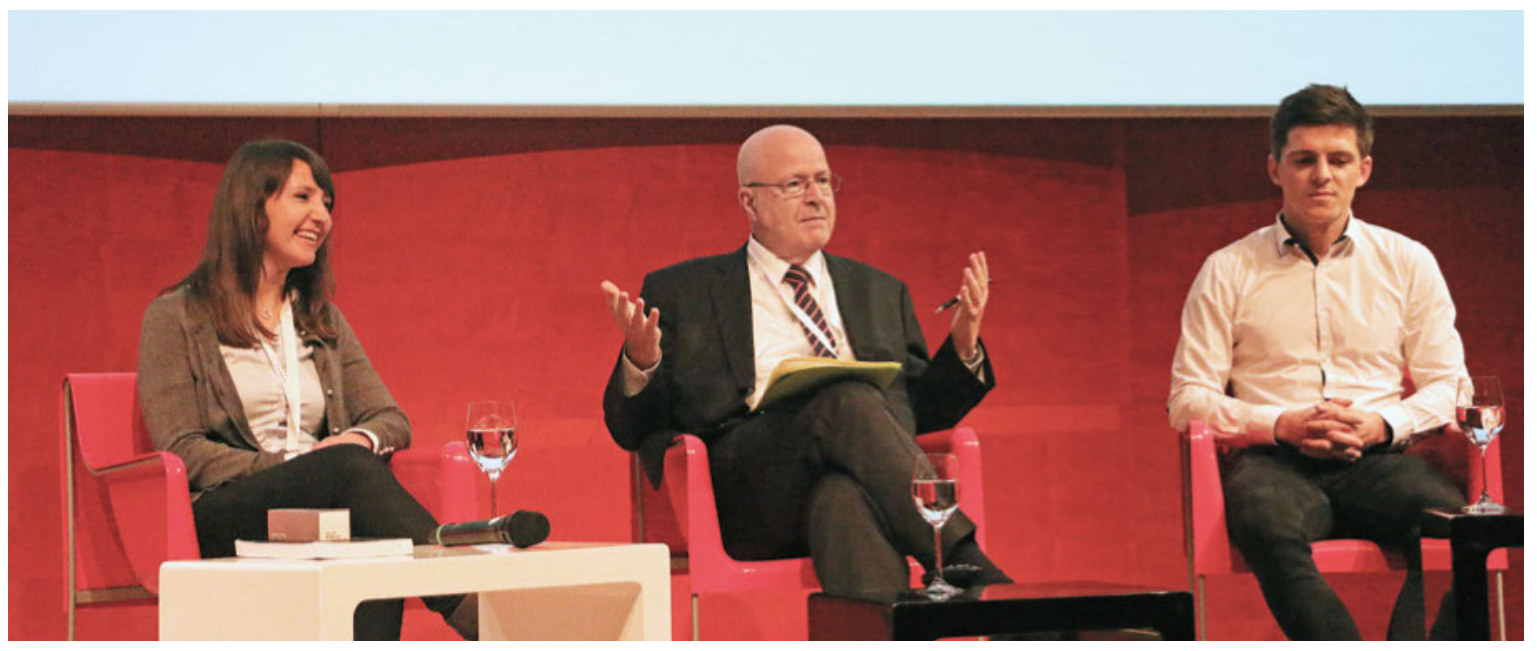

"A nous la parole»: Werner Bauer en discussion avec Livia Dülli et Rolf Erlebach, médecins en formation postgraduée. 
médecins se sont aussi montrés satisfaits du fait d'obtenir au moins quatre fois par année de tels feed-back dans le cadre des évaluations en milieu de travail. Pour eux, l'enquête annuelle d'évaluation des établissements de formation postgraduée est un outil important pour l'assurance qualité. Cet outil est d'ailleurs fréquemment utilisé par beaucoup de collègues lorsqu'il s'agit de postuler pour un nouveau poste.

\section{Une table ronde animée}

La journée s'est conclue par une table ronde animée par Iwan Rickenbacher. Avec ses invités [5], ils sont parvenus à reprendre les thèmes centraux du symposium et à les placer dans le contexte plus large de la politique de la santé. Il est notamment apparu que les experts portaient un regard différencié sur l'innovation. Henri Bounameaux s'est dit convaincu que la valeur de la relation médecin-patient restera élevée, indépendamment de l'accélération des innovations. Son collègue Bruno Schmied a souligné que l'innovation aussi doit être considérée d'un œil critique et qu'il faut soigneusement déterminer quelles innovations peuvent apporter un bénéfice, notamment dans le domaine de la formation postgraduée. Le débat a aussi été marqué par quelques déclarations intéressantes sur le thème du pilotage ou, pour rester dans le politiquement correct, de la "coordination de la formation médicale postgraduée». Certes, Stefan Spycher a évoqué les défis, mais n'a pas fondamentalement remis en question le système de formation postgraduée établi. Henri Bounameaux par contre, a estimé qu'il était tout à fait justifié de réfléchir à une réglementation. La liberté étant

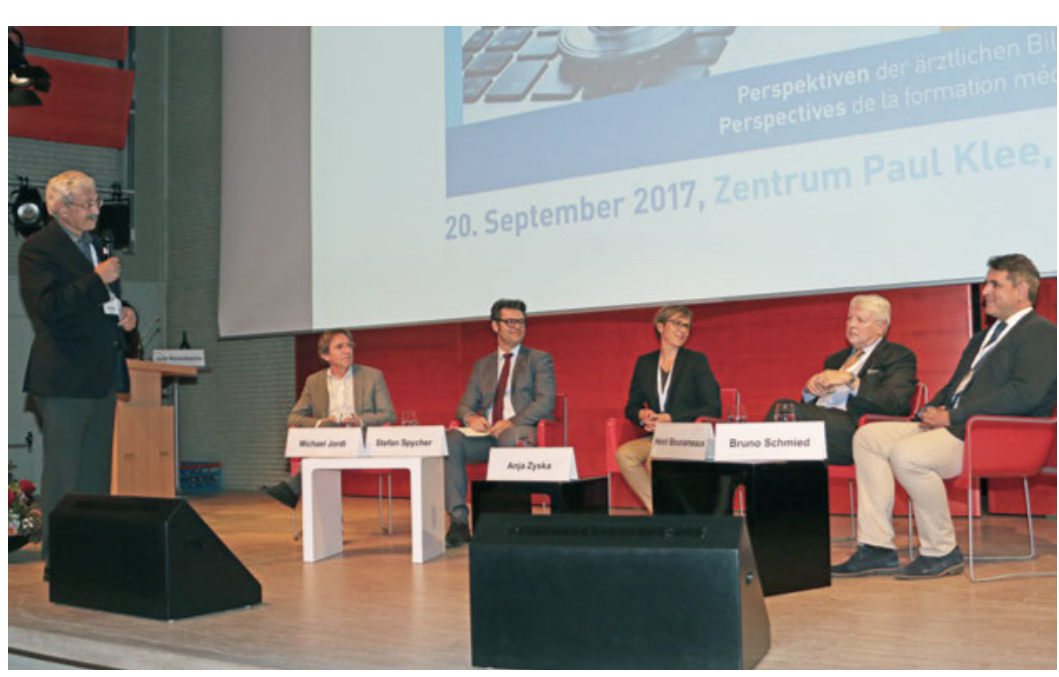

Un débat animé (de gauche à droite): Iwan Rickenbacher (animateur), Michael Jordi, Stefan Spycher, Anja Zyska, Henri Bounameaux, Bruno Schmied. bien sûr positive, mais le résultat n'étant pas toujours convaincant dans le domaine de la formation postgraduée, si l'on réfléchit au rapport entre médecins de premier recours et spécialistes. Anja Zyska a pour sa part clairement manifesté son opposition à des mesures de pilotage politiques, pour la simple raison que l'on ne dispose pas de données suffisantes à cet effet. De plus, elle a estimé qu'il était inadmissible de former des médecins et ensuite de leur refuser l'admission à pratiquer. Au niveau de la formation postgraduée, elle n'a pas exclu certaines possibilités de pilotage, mais rappelé que l'estimation des besoins pose problème. Quant à Michael Jordi, il s'est montré pragmatique, estimant qu'au lieu d'attendre de disposer de chiffres parfaits, il fallait résoudre les problèmes. A son avis, les possibilités de pilotage peuvent être envisagées d'une part pour l'admission aux études. D’autre part, les cantons devraient disposer d'outils pour intervenir lorsque la situation échappe à tout contrôle, comme c'est le cas dans les cantons de Genève, Vaud, Saint-Gall et du Tessin.

Les participants à la table ronde et la majorité du public se sont probablement ralliés à la déclaration finale d'Anja Zyska. Une formation postgraduée de qualité avec un nombre d'heures défini, comme nous la connaissons en Suisse, n'existe nulle part ailleurs. «Nous devons la préserver.» Une déclaration analogue sur le symposium MedEd ne serait probablement pas fausse. Quoi qu'il en soit, la date de l'édition 2018 peut déjà être notée: 12 septembre, au Centre Paul Klee.

\section{Crédit photos}

Bruno Kesseli

\section{Notes}

1 Les présentations des exposés et séminaires sont disponibles à l'adresse https://www.fmh.ch/fr/formation-isfm/themes/ symposium-meded/2017.html

2 Direction: Prof. Dr méd. Giatgen A. Spinas, Prof. Dr méd. Christian Lovis, Dr Karin Vey.

3 Direction: Dr méd. Regula Schmid, Prof. Dr méd., soc. dipl. Tanja Krones, Dr méd. Lukas Hegi.

4 Direction: Dr méd. Nadia Bajwa, Dr méd. Anja Zyska, Dr méd. Milena Abbiati, Dr méd. Nathalie Koch.

5 La table ronde a réuni les personnes suivantes: Prof. Iwan Rickenbacher (animation), professeur honoraire de communication politique de l'Université de Berne; Prof. Dr méd. Henri Bounameaux, doyen de la faculté de médecine de l'Université de Genève, président du Collège des Doyens, directeur de la formation et de la recherche, Hôpitaux Universitaires de Genève (HUG), Genève; lic. ès. sc. pol. Michael Jordi, secrétaire central de la Conférence suisse des directrices et directeurs cantonaux de la santé (CDS), Berne; Prof. Dr méd. Bruno Schmied, médecin-chef de la clinique de chirurgie générale, viscérale, endocrinienne et de transplantation, Saint-Gall; Dr ès sc. pol. Stefan Spycher, vicedirecteur, responsable de l'unité de direction Politique de la santé, Office fédéral de la santé publique (OFSP), Berne; Dr méd. Anja Zyska, vice-présidente, Association suisse des médecins-assistant(e)s et chef(fe)s de clinique (ASMAC), Berne. 